\title{
Anisotropic Diffusion Filter with Memory based on Speckle Statistics for Ultrasound Images
}

\author{
Gabriel Ramos-Llordén, Member, IEEE, Gonzalo Vegas-Sánchez-Ferrero, Member, IEEE, \\ Marcos Martin-Fernandez, Carlos Alberola-López, Senior Member, IEEE and Santiago Aja-Fernández
}

\begin{abstract}
Ultrasound imaging exhibits considerable difficulties for medical visual inspection and for the development of automatic analysis methods due to speckle, which negatively affects the perception of tissue boundaries and the performance of automatic segmentation methods. With the aim of alleviating the effect of speckle, many filtering techniques are usually considered as a preprocessing step prior to automatic analysis methods or visual inspection. Most of the state-of-the-art filters try to reduce the speckle effect without considering its relevance for the characterization of tissue nature. However, the speckle phenomenon is the inherent response of echo signals in tissues and can provide important features for clinical purposes. This loss of information is even magnified due to the iterative process of some speckle filters, e.g., diffusion filters, which tend to produce over-filtering because of the progressive loss of relevant information for diagnostic purposes during the diffusion process. In this work, we propose an anisotropic diffusion filter with a probabilistic-driven memory mechanism to overcome the over-filtering problem by following a tissue selective philosophy. Specifically, we formulate the memory mechanism as a delay differential equation for the diffusion tensor whose behavior depends on the statistics of the tissues, by accelerating the diffusion process in meaningless regions and including the memory effect in regions where relevant details should be preserved. Results both in synthetic and real US images support the inclusion of the probabilistic memory mechanism for maintaining clinical relevant structures, which are removed by the state-of-the-art filters.
\end{abstract}

\section{Index Terms}

Ultrasound Imaging, Speckle Filter, Anisotropic Diffusion, Memory Equations, Volterra Equations.

\section{INTRODUCTION}

$\mathbf{U}$ LTRASOUND (US) imaging has become a widely used medical diagnostic technique due to its non-invasive nature, the low cost involved and the feasibility to work in real time. Although its main drawbacks when compared to other techniques are the visual quality of the images and limitations on the field of view, it also shows several advantages, as portability and real time imaging with no emission of ionizing radiations.

US allows both diagnosis and therapeutic procedures for very different clinical purposes and specialties, such as cardiology, gynecology, neonatology, ophtalmology and orthopedics. US data are mainly used for clinical purposes in two different ways: quantitative analysis and visual inspection. Quantitative analysis involves the (manual or automatic) processing of the data in order to obtain measures that can be used for diagnosis or as biomarkers. Traditionally, these measures are related to geometric distances and volumes. An example is the assessment of nuchal translucency in obstetrics, where a distance measured over the US image of a fetus estimates the risk of Down syndrome. However, more complex indices can be considered. The strain rate of the heart quantified in 2D and 3D echocardiographies, for instance, is commonly used for the assessment of resting ventricular function or myocardial viability [1]. In the diagnosis of atherosclerosis, many works have been proposed to characterize the plaque echo-morphology in Intravascular Ultrasound (IVUS) images to identify lesions [2], [3] or to estimate the intima-media thickness of carotid artery for clinical purposes [4], [5]. In addition, US imaging provides real time in vivo visual inspection capabilities, which is customarily used by physicians to improve their diagnosis.

Both kinds of analysis, visual and quantitative, are particularly difficult tasks in US due to the presence of a characteristic granular pattern known as speckle, which reduces the contrast and the signal to noise ratio (SNR) of relevant information of tissues [6]. The low contrast and the high variability of the intensity hinder post-processing techniques such as segmentation, eliminating the possibility of using general purpose segmentation methods which may lead to erroneous conclusions or segmentation algorithms with a high lack of robustness. More sophisticated methods have been developed in the last decades to overcome this problem, e.g., active appearance models [7], level sets [8], Markov random fields [9] or Bayesian variational frameworks [10] to cite some of them. Most of the well-known segmentation methods include a filtering step prior to the segmentation process to adapt and regularize the acquired US image for the extraction of meaningful information while suppressing clinically irrelevant data [11].

The speckle pattern arises from the scattering phenomenon that takes place when US waves propagate through a biological medium [6]. The multiple reflected echoes depend on the tissue interaction of the US waves within the resolution cell. The interaction is normally modeled by using the discrete scattering theory. Particularly, the backscattering phenomenon inside the resolution cell is considered as the interaction of a discrete collection of scattering particles, scatterers, which determines the

The authors are with the Department of Teoría de la Señal y Comunicaciones e Ingeniería Telemática, University of Valladolid, 47011 Valladolid, Spain. Gonzalo Vegas-Vegas-Sánchez-Ferrero is an M+VISION Fellow at the Applied Chest Imaging Laboratory (ACIL), Brigham and Women's Hospital, Harvard Medical School, Boston, MA (e-mail: gabrll@gmail.com; gvegsan@lpi.tel.uva.es; marcma@tel.uva.es; sanaja@tel.uva.es; caralb@tel.uva.es). 
effective intensities of the reflected echoes [6]. The random location of scatterers depends on the micro structure of tissues. Therefore, the typical approach is to consider the scattering process as a random process, specifically a random walk [12], [13], which can be probabilistically modeled. This is the main reason why some authors consider speckle as noise. However, it is important to remark that the characterization of speckle as a stochastic process stems from a very different physical phenomenon than the thermal noise of other imaging modalities. In fact, the speckle pattern observed in a quiescent region provides the same granular pattern [14]. Consequently, speckle is tissue-dependent and it is useful for characterizing different tissues [15], [16], [2], [4], [5]. Traditionally, it has been modeled by taking into account the signal received at the transducer, disregarding the further processing steps. The envelope of the received signal, i.e., the envelope of the complex random walk, can be probabilistically classified into four speckle models, depending on the number of effective scatterers $\left(N_{\text {eff }}\right)$ considered within the resolution cell. Fully developed speckle arises when $N_{\text {eff }}$ is high. In this scenario, the probabilistic distribution of the envelope is Rayleigh. This model usually holds in blood regions. If the random walk additionally possesses a deterministic component, the model is known as fully resolved and the Rayleigh distribution is replaced by a Rician distribution. Regions with high echolucent response such as myocardium tend to produce this kind of speckle. When the condition of high $N_{\text {eff }}$ is not satisfied, the resulting models are known as partially developed and partially resolved speckle and the probability density functions follow the $K$ distribution and the Homodyned $K$ distribution respectively [17].

Although in practice fully developed and fully resolved speckle are the most common, the empirical distributions obtained from the US images deviate from them due to signal post-processing steps [18]. Consequently, some attempts have been made to model the empirical distributions with useful probabilistic models, e.g., Nakagami, Gamma or Weibull [19], [18], [5], [20], [21].

Since speckle is one of the limiting factors in the final quality of US images, many US speckle-removing filters have been proposed in the last two decades. Those filters consider speckle as an undesired noise and try to remove it while preserving the edges of relevant tissues. This treatment of speckle needs to be carefully taken since it may also provide useful medical information in certain type of tissues [22], which has been favorably used in tissue characterization [2], [4], [5]. In the early contributions, speckle US filters were inspired by the filters developed in the synthetic aperture radar (SAR) field because of the similarities observed in both modalities [12]. However, the characterization of speckle was not incorporated into the filtering methods and therefore they tend to remove the speckle texture of tissues, blur edges and, in some cases, suppress small but informative tissue details. Some of them (chronologically cited) are the Lee filter [23], the Frost filter [24], the improved Lee version due to Kuan [25] or the Gamma MAP (maximum a posteriori) filter [26]. We will refer to these filters as the classical speckle filters. They rely on a multiplicative signal model which establishes the relationship of the statistics of the random image with the tissues, where noise was commonly considered Gaussian distributed. Because of the inaccurate speckle statistical modeling, they tend to remove important details.

More elaborated filters try to overcome the problem of poor definition of edges by means of the non-homogeneous diffusive phenomenon of heat. Such is the case of the celebrated speckle reducing anisotropic diffusion filter (SRAD) [27] or improved approaches such as the detail preserving anisotropic diffusion filter (DPAD) [28] and its oriented tensor extension, oriented speckle reducing anisotropic diffusion filter (OSRAD) [29]. All these filters rely on the multiplicative noise model and, as in the case of classical speckle filters, the speckle pattern in tissues is removed when the diffusion process evolves, hence generating nearly piece-wise constant images. A more refined approach of diffusion filters is the so-called POSRAD, i.e., probability-driven OSRAD [18], which accounts for the statistical models of tissues and provides better results than the above mentioned filters, though the structural elimination of details is still intrinsically present due to the nature of the diffusion equation.

A different paradigm was used with the non local means method applied to speckle denoising, the so-called optimized Bayesian non local means (OBNLM) [30]. However, the assumption of local regions of homogeneity in US medical images is controversial and, if not appropriate, it may even enhance the speckle in those regions.

All in all, the main problems of US filtering can be summarized as: 1) The relevant information is not accurately characterized. 2) Even with an accurate characterization, diffusion filters suffer from over-filtering due to the loss of original information during the diffusion process [31].

Considering these issues and the limitations of the state-of-the-art filters, our contribution is the development of a speckle anisotropic diffusion filter based on realistic probabilistic models with a memory mechanism to preserve relevant information during the filtering process. The memory mechanism, implemented as a delay differential equation for the diffusion tensor, adaptively preserves medical details encoded in the speckle probabilistic characterization of tissues and efficiently removes speckle in clinical meaningless regions.

The paper is structured as follows: In section II a comprehensive description of the above mentioned speckle filters is addressed. The proposal of the method is presented in section III and implementation details are described in section IV. In section $\mathrm{V}$ both real and synthetic experiments are carried out by comparing the method with the previously cited speckle filters. Conclusions are summarized in section VI. 


\section{BACKGROUND}

\section{A. Classical Filters}

As previously stated in the introduction, many filters have been developed to cope with speckle, with differences lying on the assumptions about the speckle model, where the multiplicative model is the one mostly used. Among the vast amount of approaches, we will highlight the most relevant.

First approaches in speckle filtering assumed the noise to be multiplicative where the observable image, $g(\mathbf{x})$, is described as:

$$
g(\mathbf{x})=f(\mathbf{x}) n(\mathbf{x}),
$$

where $f$ is the "true" image, $n$ is a noise perturbation and $\mathbf{x} \in \mathbb{R}^{3}$ is the spatial location. One of the first contribution is due to Lee [23], where authors assume that the noise is Gaussian distributed with unitary mean and constant standard deviation $\sigma_{n}$. The image $f$ is then estimated using a local linear minimum mean-square estimator (LLMMSE). Kuan et al. [25] developed a similar expression for the estimation of $f$, but avoiding the Taylor approximation done in the previous filter, and thus providing the exact optimal estimate according to the LLMMSE criterion. A similar probabilistic approach was done by Frost et al. [24] but using a minimum mean-square error criterion. A reformulation of Lee's and Kuan's approaches as a maximum a posteriori (MAP) estimation was proposed by Lopes et al. [26], in what they called the Gamma MAP filter.

\section{B. Diffusion Filters for Speckle}

A entirely different paradigm about speckle filtering started with the application of anisotropic diffusion techniques in this field by Yu and Acton [27]. Diffusion techniques in image filtering are based on the well-known diffusion equation [31]:

$$
\frac{\partial u(\mathbf{x}, t)}{\partial t}=\nabla \cdot(\mathbf{D} \nabla u(\mathbf{x}, t))
$$

with initial condition $u_{0}(\mathbf{x})=u(\mathbf{x}, t=0)$. $\mathbf{D}$ is a symmetric positive definite tensor which depends on the local structure of $u$ and $\nabla \cdot$ and $\nabla$ denote the divergence and gradient operators respectively. In this case, $u(\mathbf{x}, 0)$ is the noisy image and $t$ denotes the temporal variable. If $\mathbf{D}$ reduces to a scalar function, the filter is usually termed as isotropic non-homogeneous diffusion filter. On the other hand, in the tensorial case, the term anisotropic diffusion filter is usually adopted [31]. Most of diffusion filters are modifications of the Perona-Malik filter [32], where D is a scalar coefficient based on the gradient of the image $c(\nabla u)$ which avoids diffusion near the boundaries and applies it in homogeneous areas.

Yu and Acton [27] established a link between Lee's filter and the discrete implementation of the Perona-Malik filter which motivates them to propose the SRAD filter, formulated as follows:

$$
\begin{aligned}
& \frac{\partial u(\mathbf{x}, t)}{\partial t}=\nabla \cdot(c(q) \nabla u(\mathbf{x}, t)), \\
& u(\mathbf{x}, t=0)=u_{0}(\mathbf{x}),\left.\quad \frac{\partial u(\mathbf{x}, t)}{\partial \hat{n}}\right|_{\partial \Omega}=0
\end{aligned}
$$

where the coefficient of diffusion is defined as a decreasing function of the instantaneous coefficient of variation. The method needs the estimate of a parameter $q_{0}(t)$ related to the coefficient of variation of noise. The estimation of this parameter is precisely the weakest point of the method, as was shown in [28], where the authors proposed an improved version of the SRAD named the detail preserving anisotropic diffusion (DPAD). The DPAD filter relies on Kuan's filter (instead of Lee's) and mostly decouples the problem of noise estimation and image filtering, by using different window sizes for each task. Authors showed, by using improved noise estimators, that DPAD and SRAD may provide similar results, thus highlighting the importance of a proper noise estimation.

These two approaches cannot be accurately considered to be anisotropic, since they both use a scalar value (and not a tensor) as a diffusion function ${ }^{1}$. On the other hand, the extension proposed in [29] by the OSRAD filter is properly an anisotropic approach. The method uses a tensor $\mathbf{D}$ which is diagonalized with eigenvalues $\lambda_{1}, \lambda_{2}$ and $\lambda_{3}$ and eigenvectors $\mathbf{v}_{\mathbf{1}}, \mathbf{v}_{\mathbf{2}}$ and $\mathbf{v}_{\mathbf{3}}$, respectively. The authors select $\mathbf{v}_{\mathbf{1}}$ as the direction of the gradient and $\mathbf{v}_{\mathbf{2}}$ and $\mathbf{v}_{\mathbf{3}}$ being the maximal and minimal curvature directions computed on the Gaussian smoothed image. These directions are acquired as the eigenvectors of the projection of the Hessian matrix $\mathbf{H}$, in the plane orthogonal to the gradient. Along the direction of $\mathbf{v}_{\mathbf{1}}$, the filter should detect the presence of an edge to apply smoothing or to preserve the boundaries. Krissian et al. define $\lambda_{1}$ as $1-k_{\text {Kuan }}(\mathbf{x})$ and fixed $\lambda_{2}$ and $\lambda_{3}$ to constant $c_{\max }$ and $c_{\min }$ with $c_{\max } \gg c_{\min }>0$. Consequently, the filtered image with OSRAD filter is the solution $u$ of Eq. (2) with

$$
\mathbf{D}=\left(\mathbf{v}_{\mathbf{1}}\left|\mathbf{v}_{\mathbf{2}}\right| \mathbf{v}_{\mathbf{3}}\right)\left(\begin{array}{ccc}
1-k_{\text {Kuan }}(\mathbf{x}) & 0 & 0 \\
0 & c_{\max } & 0 \\
0 & 0 & c_{\min }
\end{array}\right)\left(\begin{array}{c}
\mathbf{v}_{\mathbf{1}}^{T} \\
\mathbf{v}_{\mathbf{2}}^{T} \\
\mathbf{v}_{\mathbf{3}}^{T}
\end{array}\right)
$$

\footnotetext{
${ }^{1}$ Note that in this manuscript the term anisotropic diffusion follows the terminology commonly adopted in physics and mathematics, where the anisotropy is necessarily described by the diffusion tensor. For an interesting discussion on inhomogeneous diffusion vs. anisotropic diffusion we refer the reader to [31].
} 
In case of 2D images, the parameters $c_{\max }$ and $c_{\min }$ reduce to just one coefficient, denoted as $c_{\text {tang }}$.

The key of a proper filtering in the OSRAD approach depends mainly on the definition of the diffusion tensor $\mathbf{D}$. To improve the accuracy of the method, Vegas-Sánchez-Ferrero et al. proposed in [18] the POSRAD filter, and extension of the OSRAD approach which uses a probabilistic framework to better define the diffusion tensor $\mathbf{D}$. The intensity of the image $X$ is modeled as a random variable that follows a J-component mixture of distributions,

$$
f_{X}\left(x ; \pi_{1}, . ., \pi_{J}, \boldsymbol{\theta}_{1}, \ldots, \boldsymbol{\theta}_{J}\right)=\sum_{j=1}^{J} \pi_{j} f_{X_{j}}\left(x ; \boldsymbol{\theta}_{j}\right),
$$

where $\pi_{j}>0, \sum_{j=1}^{J} \pi_{j}=1$ and $f_{X_{j}}$ are probability density functions with parameters vector $\boldsymbol{\theta}_{j}$. The probability that a pixel with intensity $x$ belongs to the tissue class $C_{j}, P\left(x \in C_{j} \mid X=x ; \boldsymbol{\theta}_{j}\right)$, was obtained by means of the Bayes Theorem.

The authors define a structure tensor for each class $C_{j}$ as the Weickert's local structure tensor [31],

$$
\mathbf{T}_{j}=G_{\sigma} * \nabla_{\sigma}\left(P\left(x \in C_{j} \mid X=x ; \boldsymbol{\theta}_{j}\right) \nabla_{\sigma} P\left(x \in C_{j} \mid X=x ; \boldsymbol{\theta}_{j}\right)^{T}\right.
$$

where $*$ denotes spatial convolution.

The diffusion tensor $\mathbf{D}$ is diagonalized with eigenbase $\left\{\mathbf{e}_{\mathbf{1}}, \mathbf{e}_{\mathbf{2}}, \mathbf{e}_{\mathbf{3}}\right\}$ obtained from the $\hat{j}^{\text {th }}$ structure tensor $\mathbf{T}_{\hat{j}}$ whose maximum eigenvalue, $\mu_{1}^{j}$, is maximal for all $j$, i.e., $\hat{j}=\arg \max _{j} \mu_{1}^{j}$. Therefore, the most probable boundary is preserved during the filtering process. The eigenvalues $\lambda_{1}, \lambda_{2}$ and $\lambda_{3}$ are defined as,

$$
\begin{aligned}
& \lambda_{1}=1-\left|\nabla_{\sigma} P\left(x \in C_{\hat{j}} \mid X=x ; \boldsymbol{\theta}_{\hat{j}}\right) \cdot \mathbf{e}_{1}\right|, \\
& \lambda_{2}=1-\left|\nabla_{\sigma} P\left(x \in C_{\hat{j}} \mid X=x ; \boldsymbol{\theta}_{\hat{j}}\right) \cdot \mathbf{e}_{\mathbf{2}}\right|, \\
& \lambda_{3}=1 .
\end{aligned}
$$

In homogeneous areas, $\mathbf{D}$ becomes isotropic and in the presence of boundaries it becomes anisotropic with the main orientation aligned along the most probable boundaries. The least eigenvalue, $\lambda_{3}$, is defined in this way to perform the filtering process along the direction of the least probable boundary.

\section{Non Local Means Filters for Speckle}

Another entirely different approach is based on the non local means (NLM) filter firstly proposed by Buades et al. [33] for general image denoising applications. In this methodology, the value in a single voxel of the output image $g$ is obtained by a weighted average of the intensities of different voxels in the neighborhood. The different weights are calculated as a function of the similarity between values and the distance to the voxel.

This approach has been applied to US with the so-called optimized Bayesian NLM (OBNLM) proposed by Coupé et al. [30]. In their work, in addition to conceiving an optimized version of the NLM using a block-wise approach, they proposed a Pearson distance instead of the Euclidean norm based on the probabilistic model:

$$
g(\mathbf{x})=f(\mathbf{x})+\sqrt{f(\mathbf{x})} n(\mathbf{x}),
$$

where $n$ is Gaussian noise with zero mean and constant standard deviation $\sigma_{n}$.

\section{Volterra Filter}

Cottet and El Ayyadi [34] proposed an anisotropic nonlinear diffusion equation which was combined with a time-delay regularization of partial differential equations. Specifically and following their notation, the filtered image is the solution of the following system of equations:

$$
\begin{aligned}
& \frac{\partial u(\mathbf{x}, t)}{\partial t}=\nabla \cdot(\mathbf{L}(\mathbf{x}, t) \nabla u(\mathbf{x}, t)) \\
& \frac{\partial \mathbf{L}(\mathbf{x}, t)}{\partial t}+\frac{\mathbf{L}(\mathbf{x}, t)}{\tau}=\frac{\mathbf{F}\left(\nabla_{\sigma} u(\mathbf{x}, t)\right)}{\tau}
\end{aligned}
$$

where $\mathbf{L}(\mathbf{x}, t)$ is the diffusion tensor at point $\mathbf{x}$ and time $t$. Note the temporal dependence due to the coupling with the delay differential equation (DDE), Eq. (10). The initial conditions are the noisy image, $u_{0}(\mathbf{x})$, the initial diffusion tensor $\mathbf{L}(\mathbf{x})$ and the periodic boundary conditions [34]. With this formulation, the selection of a stopping time is avoided because the filtered image is considered as the steady solution of the following Volterra equation:

$$
\frac{\partial u(\mathbf{x}, t)}{\partial t}-e^{-\frac{t}{\tau}} \nabla \cdot\left(\mathbf{L}(\mathbf{x}, 0) \nabla_{\sigma} u(\mathbf{x}, t)\right)
$$

$$
-\int_{0}^{t} e^{\frac{s-t}{\tau}} \nabla \cdot\left(\mathbf{F}\left(\nabla_{\sigma} u(\mathbf{x}, s)\right)\left(\nabla_{\sigma} u(\mathbf{x}, t)\right) d s=0 .\right.
$$


Cottet and El Ayyadi related the relaxation time $\tau$ with a minimum scale parameter. They defined the application $\mathbf{F}$ by projecting higher gradients into the direction of edge contours and performing isotropic diffusion otherwise.

Strictly speaking, the Volterra filter is not a speckle filter. The method we propose is inspired by the Volterra equation proposed by Cottet and El Ayyadi. In our method, we extend the memory mechanism of the Cottet and El Ayyadi filter to a probabilistic memory mechanism which is tissue selective.

\section{Proposed Method}

\section{A. Anisotropic Diffusion Filter with Memory based on Speckle Statistics}

The philosophy of [34] is adapted to the US medical imaging context. The main novelty beyond [34] philosophy is to avoid the influence of gradient information due to the lack of contours and low contrast of US images by means of a probabilisticdriven selective filtering that preserves relevant clinical details in regions of interest due to the effect of the memory equations. Two different mechanisms are formulated to take advantage of the tissue characterization with the aim of preserving relevant clinical information: First, we include a selective diffusion mechanism by means of the probabilistic characterization of tissues in US images. Second and more important, we extend the formulation of [34] to a probability-driven memory mechanism in order to establish different memory behaviors depending on tissues. The first one leads to a more aggressive filtering in regions with no relevant information for clinical purposes, whereas the diffusion in regions with presumable relevant information (regions with structures and textures used for diagnosis) is reduced. The second one accounts for the preservation of the structures through the diffusion process in regions of interest by leading to steady states where the undesired speckle has been removed in non relevant regions and the important structures remain visible.

Both extensions considerably alleviate the problem of over-filtering present in the speckle diffusion filter paradigm because information from the beginning of the process is always taken into account in further iterations. The selective memory mechanism is established by introducing a spatial dependence on the relaxation time $\tau$ and by defining a new filtering tensor operator $\mathcal{S}\{$.$\} that accounts for the selective preservation of tissue (the latter will be explained in detail in a further section).$ These are the main differences compared to the original version proposed by Cottet and El Ayyadi, which leads to a new physical interpretation of the filtering process.

Our proposed system of coupled equations is the following:

$$
\begin{aligned}
& \frac{\partial u(\mathbf{x}, t)}{\partial t}=\nabla \cdot(\mathbf{L}(\mathbf{x}, t) \nabla u(\mathbf{x}, t)), \\
& \frac{\partial \mathbf{L}(\mathbf{x}, t)}{\partial t}=\frac{1}{\tau(\mathbf{x})}(\mathbf{L}(\mathbf{x}, t)-\mathcal{S}\{\mathbf{D}(\mathbf{x}, t)\}),
\end{aligned}
$$

with the same initial and periodic conditions as in the formulation of Cottet and El Ayyadi filter. The tensor $\mathbf{D}$ is the instantaneous diffusion tensor. For the sake of readability, the spatial dependence of $\tau$ will be avoided hereafter. The DDE of Eq. (13) can be easily integrated as it was done in [34]:

$$
\mathbf{L}(\mathbf{x}, t)=\mathcal{S}\{\mathbf{D}(\mathbf{x}, 0)\} e^{\frac{-t}{\tau}}+\int_{0}^{t} e^{\frac{s-t}{\tau}} \mathcal{S}\{\mathbf{D}(\mathbf{x}, s)\} d s .
$$

An important consequence of the proposed new system of equations is that the whole diffusion process cannot be directly formulated as a Volterra equation as in Eq. (11) because of the spatial dependence of $\tau$. Instead, our model has to be formulated as a Volterra equation in the sense of the diffusion flux $\mathbf{f}(\mathbf{x}, t)=\mathbf{L}(\mathbf{x}, t) \nabla u(\mathbf{x}, t)$, that can be written as:

$$
\mathbf{f}(\mathbf{x}, t)=\overbrace{e^{\frac{-t}{\tau}} \mathcal{S}\{\mathbf{D}(\mathbf{x}, 0)\} \nabla u(\mathbf{x}, t)}^{\mathbf{f}_{1}(\mathbf{x}, t)}+\overbrace{\int_{0}^{t} e^{\frac{s-t}{\tau}} \mathcal{S}\{\mathbf{D}(\mathbf{x}, s)\} \nabla u(\mathbf{x}, t) d s}^{\mathbf{f}_{2}(\mathbf{x}, t)} .
$$

Let us define $\mathbf{f}^{t, s}$ as the diffusion flux at time $t$ with filtered diffusion tensor $\mathcal{S}\{\mathbf{D}(\mathbf{x}, s)\}, s \leqslant t$, i.e.:

$$
\mathbf{f}^{t, s}(\mathbf{x})=\mathcal{S}\{\mathbf{D}(\mathbf{x}, s)\} \nabla u(\mathbf{x}, t) .
$$

The diffusion flux can be written in terms of $\mathbf{f}^{t, s}(\mathbf{x})$ as:

$$
\begin{aligned}
& \mathbf{f}_{1}(\mathbf{x}, t)=e^{\frac{-t}{\tau}} \mathcal{S}\{\mathbf{D}(\mathbf{x}, 0)\} \nabla u(\mathbf{x}, t)=e^{\frac{-t}{\tau}} \mathbf{f}^{t, 0} \\
& \mathbf{f}_{2}(\mathbf{x}, t)=\int_{0}^{t} e^{\frac{s-t}{\tau}} \mathcal{S}\{\mathbf{D}(\mathbf{x}, s)\} \nabla u(\mathbf{x}, t) d s=\int_{0}^{t} e^{\frac{s-t}{\tau}} \mathbf{f}^{t, s} d s .
\end{aligned}
$$

Note that the diffusion direction at $t$ is a weighted average in the temporal window $[0, t]$ of the flux directions $\mathbf{f}^{t, s}$ with increasing weights (the more recent fluxes, the more relevant) and the contribution of $\mathbf{f}^{t, 0}$ decays exponentially as time $t$ increases. Therefore, as it was expected, the filtering process is performed with a memory behavior because, at time $t$, the diffusion directions are calculated considering the structural information of previous times. In terms of diffusion fluxes, the 


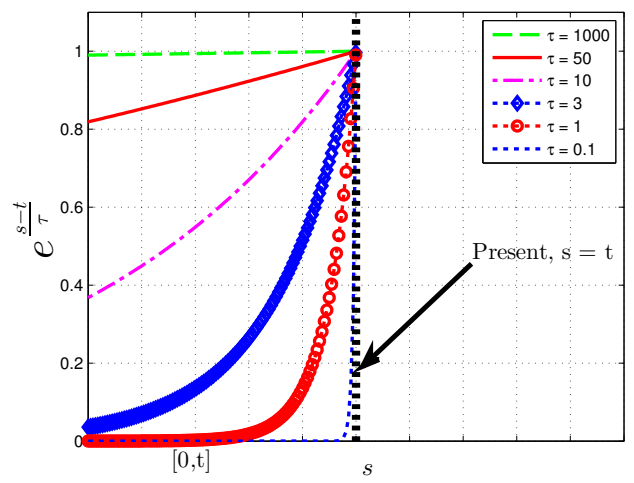

Fig. 1. Effective temporal window for different values of $\tau$. The curves represent the memory effect of the weighting function $\exp \left(\frac{s-t}{\tau}\right)$ in the weighted average of flux directions $\mathbf{f}^{t, s}$. Note that, for small values of $\tau$, the filtering is performed without memory. Conversely, the memory is strongly preserved as $\tau$ increases.

applied flux comes from two different sources, each one considering the structural information of that region of the image at different time. The first flux "predicts" the instantaneous structural information by simply propagating the initial information of the noisy image, i.e., by propagating the initial diffusion directions to the time $t$. This contribution exponentially vanishes depending on the relaxation time. The second flux produces a more refined "prediction" because it is governed by the temporal history of the diffusion tensor at one specific region. Therefore, the effect of aggressive decisions in the filtering process is mitigated thanks to this temporal average.

This fact is exploited to develop the already mentioned tissue selective scheme by varying $\tau(\mathbf{x})$ according to the tissue characterization.

The local effect of $\tau$ in the memory mechanism is the following:

- When $\tau \rightarrow 0$, no memory is locally applied. $\mathbf{f}(\mathbf{x}, t) \approx \mathbf{f}^{t, t}=\mathcal{S}\{\mathbf{D}(\mathbf{x}, t)\} \nabla u(\mathbf{x}, t)$. This can be rigorously demonstrated when $\frac{\partial}{\partial t} \mathbf{L}(\mathbf{x}, \mathbf{t})$ is bounded ${ }^{2}$, then $\lim _{\tau \rightarrow 0}(\mathcal{S}\{\mathbf{D}(\mathbf{x}, t)\}-\mathbf{L}(\mathbf{x}, \mathbf{t}))=\lim _{\tau \rightarrow 0} \tau \frac{\partial}{\partial t} \mathbf{L}(\mathbf{x}, \mathbf{t})=0$

- When $\tau \rightarrow \infty$, all the information is preserved. The weights $e^{\frac{s-t}{\tau}}$ become nearly constant, so all the information is equally weighted and the memory mechanism preserves all the information of previous times.

Intermediate values of $\tau$ are related to an intermediate memory state. Thus, the relaxation time can be thought of as an "effective" temporal window in contrast to considering the whole interval $[0, t]$ equally. The behavior of the "effective" temporal window is shown in Fig. 1 for different values.

In regions with relevant information, the temporal window must be large, while meaningless regions should use a smaller effective window, just accounting for instantaneous information.

The formulation provided in Eqs. (12) and (13) allows even a more refined preservation of relevant structures by means of a suitable definition of the filtering tensor operator $\mathcal{S}\{$.$\} , undefined so far. The following section is devoted to its definition$ and the definition of $\tau(\mathbf{x})$.

\section{B. Selective memory: Definition of $\tau(\mathbf{x})$ and $\mathcal{S}\{$.}

The selective memory according to different tissues can be accomplished by a suitable tissue characterization. We decide to use a probabilistic characterization based on the speckle statistics already motivated in the introductory section. Both the relaxation time $\tau$ and the operator $\mathcal{S}\{$.$\} become tissue selective when the information provided by the probability of belonging$ to some specific tissue is considered. In this work, we show on the simplest case, i.e., $\tau$ and $\mathcal{S}\{$.$\} only depend on one probability$ map, though the formulation presented in the previous section is not confined to this scenario.

Note that, in this scenario, both expressions may depend on $p_{\bar{c}}(\mathbf{x}, t)$, representing the probability of the regions considered as meaningless or $p_{c}(\mathbf{x}, t)=1-p_{\bar{c}}(\mathbf{x}, t)$, standing for the probability of the tissues to be preserved.

The filter should reach stationary states $\left(\frac{\partial}{\partial t} u(\mathbf{x}, t)=0\right)$ in relevant regions, where the filter does not evolve and the structures are maintained. This can be accomplished by defining the filtering tensor operator $\mathcal{S}\{$.$\} as a transformation of the instantaneous$ diffusion tensor at locations $\mathbf{x}$, where preservation must be performed, into a null tensor. One simple way to include this selective behavior in the definition of the diffusion tensor is by multiplying its eigenvalues by $p_{\bar{c}}(\mathbf{x}, t)$ and reconstructing again the tensor by using the outer product expansion:

$$
\mathcal{S}\{\mathbf{D}(\mathbf{x}, t)\}=\mathbf{E}(\mathbf{x}, t) \mathbf{\Sigma} \mathbf{E}(\mathbf{x}, t)^{T},
$$

where

$$
\boldsymbol{\Sigma}=p_{\bar{c}}(\mathbf{x}, t)\left(\begin{array}{ccc}
\lambda_{1}(\mathbf{x}, t) & 0 & 0 \\
0 & \lambda_{2}(\mathbf{x}, t) & 0 \\
0 & 0 & \lambda_{3}(\mathbf{x}, t)
\end{array}\right)
$$

\footnotetext{
${ }^{2}$ Due to the high temporal resolution of US images, one can fairly assume that no abrupt temporal changes exist.
} 


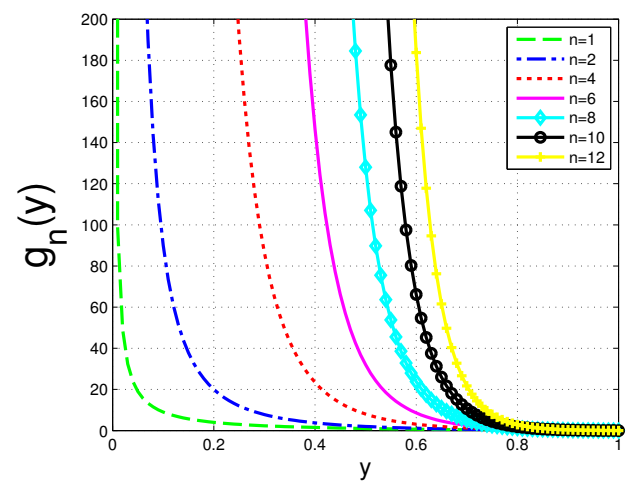

Fig. 2. Behavior of the family of rational functions $g_{n}(y)$ depending on $n$. When $n$ increases, only regions with a high value of $y$ are not affected by the memory mechanisms. Therefore, the filter tends to be more conservative.

and $\mathbf{E}=\left(\mathbf{e}_{1}\left|\mathbf{e}_{2}\right| \mathbf{e}_{3}\right)$. The eigenvectors $\mathbf{e}_{i}$ and eigenvalues $\lambda_{i}(\mathbf{x}, t)$ with $i=\{1,2,3\}$ are obtained following the approach of POSRAD.

Notice that the operator $\mathcal{S}\{$.$\} is not directly applied to \mathbf{L}(\mathbf{x}, t)$ but it is included in the linear Volterra equation. The reason for this is to keep track of the time-dependent probability to get a more robust characterization than that obtained from the instantaneous probability $p_{\bar{c}}(\mathbf{x}, t)$. Therefore, accounting for the "historical" probability of meaningless information provides more robust characterization than the instantaneous probability.

For the relaxation time, $\tau$, we propose to model it as a one-dimensional function of $p_{\bar{c}}(\mathbf{x}, t)$, i.e., $\tau=\tau\left(p_{\bar{c}}(\mathbf{x}, t)\right)$. The function $\tau:[0,1] \longmapsto(0, \infty)$ should meet some requirements in order to turn on/off the memory mechanism depending on the regions and tissues:

1) Maximum memory for relevant tissues. When $p_{\bar{c}}(\mathbf{x}, t) \rightarrow 0$, the memory mechanism must account for all the historical information about the diffusion directions. Therefore, $\tau\left(p_{\bar{c}}(\mathbf{x}, t)\right) \rightarrow \infty$.

2) Memory suppression in meaningless regions. As $p_{\bar{c}}(\mathbf{x}, t) \rightarrow 1$, the memory should tend to disappear. Then, $\tau\left(p_{\bar{c}}(\mathbf{x}, t)\right) \rightarrow$ 0.

3) Continuous and monotonically increasing function. This restriction is imposed to provide a smooth memory behavior, since continuous spatial variation of the probability maps should provide continuous memory preservation of details.

The range of functions for which these properties hold is very broad. We have considered the family of rational functions of one parameter $n \geqslant 1$ defined as:

$$
g_{n}(y)=\frac{1-y}{y^{n}}, \text { with } y>0 .
$$

This family of functions fulfills the above mentioned requirements and provide a practical implementation due to its oneparameter definition. They are also infinitely derivable, which is an important property to provide stability in the numerical implementation scheme. This family of functions is shown in Fig. 2 for different values of $n$. Note that the parameter $n$ can be considered as a measure of the conservative behavior of the memory mechanism. For a constant abscissa $y$, the higher $n$, the more conservative the filter. However, the constraint $g_{n}(1)=0$ imposed by definition ensures that in meaningless regions (where, $p_{\bar{c}}(\mathbf{x}, t) \rightarrow 1$ ) the memory mechanism is suppressed. In fact, the family of functions $g_{n}$ allows to recreate a threshold behavior with the benefits of the smoothness and regularity properties. In Fig. 2 one can clearly see the effect of $n$ for $\tau=g_{n}\left(p_{\bar{c}}(\mathbf{x}, t)\right)$ : when $n$ increases, only regions with a high value of $p_{\bar{c}}(\mathbf{x}, t)$ are not affected by the memory mechanism. Therefore, the filter tends to be more conservative and only clearly meaningless regions turn the memory off, i.e when $p_{\bar{c}}(\mathbf{x}, t) \rightarrow 1$.

\section{IMPLEMENTATION}

\section{A. The speckle characterization of $p_{\bar{c}}(\mathbf{x}, t)$}

The probabilistic characterization required in the selective memory mechanism is based on speckle probability distributions. In this work, we make use of the Gamma distribution since it has shown its suitability to model fully developed and resolved speckle after interpolating/linear filtering steps [5], [35]. Consequently, the diffusion tensor $\mathbf{D}(\mathbf{x}, t)$, which is implemented as in POSRAD [18], is obtained from the mixture of density probability functions, Eq. (5), whose components are Gamma density functions. We refer to this model as Gamma Mixture Model (GMM). Note that the method we propose is independent of the distributions used for its characterization and any other distribution such as the Nakagami or Rayleigh can be used.

In our experiments, we consider the blood regions as the meaningless information regions. The class for the blood region was selected as that with least mean value, since the echo-lucent response is generally lower than in tissue regions [19]. Then, $p_{\bar{c}}(\mathbf{x}, t)$ is obtained as the a posteriori probability of that class. The parameters of the GMM are estimated by maximizing the log-likelihood function with the Expectation-Maximization algorithm [36], [21], [5]. 


\section{B. Discretization}

Our system of coupled equations was discretized with the semi-implicit method of Weickert for the anisotropic diffusion [37] and, in a similar way as in [34], we calculate $\mathbf{L}^{n+1}$ as:

$$
\mathbf{L}^{n+1}=\frac{1}{1+\beta^{n}}\left(\beta^{n} \mathbf{L}^{n}+\mathcal{S}^{n}\{\mathbf{D}\}\right)
$$

where $\beta^{n}=\frac{\tau\left(\mathbf{x}, n \Delta_{t}\right)}{\Delta_{t}}$ and $\mathbf{L}^{n+1}, \mathbf{F}^{n}$ denote the updated tensor and the tensor obtained from the image $u^{n}$ at iteration $n+1$ and $n$ respectively. This implementation has good stability properties. In fact, with the assumptions that no abrupt direction changes happen, the eigenvectors of $\mathbf{L}^{n+1}, \mathbf{L}^{n}$ and $\mathcal{S}^{n}\{\mathbf{D}\}$ are similar and, by means of the diagonalization of Eq. (22), the following update recursion for the eigenvectors of $\mathbf{L}^{n+1}, \gamma_{1}^{n+1}, \gamma_{2}^{n+1}$ and $\gamma_{3}^{n+1}$ can be derived:

$$
\left(\begin{array}{c}
\gamma_{1}^{n+1} \\
\gamma_{2}^{n+1} \\
\gamma_{3}^{n+1}
\end{array}\right)=\left(\begin{array}{ccc}
\frac{\beta^{n}}{1+\beta^{n}} & 0 & 0 \\
0 & \frac{\beta^{n}}{1+\beta^{n}} & 0 \\
0 & 0 & \frac{\beta^{n}}{1+\beta^{n}}
\end{array}\right)\left(\begin{array}{c}
\gamma_{1}^{n} \\
\gamma_{2}^{n} \\
\gamma_{3}^{n}
\end{array}\right)+\frac{1}{1+\beta^{n}}\left(\begin{array}{c}
\lambda_{1}^{n} \\
\lambda_{2}^{n} \\
\lambda_{3}^{n}
\end{array}\right)
$$

Note that $\gamma_{1}^{n+1}, \gamma_{2}^{n+1}$ and $\gamma_{3}^{n+1}$ are always non negative because $\beta^{n}, \gamma_{1}^{n}, \gamma_{2}^{n}, \gamma_{3}^{n}, \lambda_{1}^{n}, \lambda_{2}^{n}$ and $\lambda_{3}^{n}$ are non negative. Hence, the updated tensor is non negative definite. Finally, the tensor can be reconstructed from the eigenvectors of either $\mathbf{L}^{n}$ or $\mathcal{S}^{n}\{\mathbf{D}\}$ with the outer expansion formula:

$$
\mathbf{L}^{n+1}=\gamma_{1}^{n+1} \mathbf{e}_{1} \mathbf{e}_{1}^{T}+\gamma_{2}^{n+1} \mathbf{e}_{2} \mathbf{e}_{2}^{T}+\gamma_{3}^{n+1} \mathbf{e}_{3} \mathbf{e}_{3}^{T}
$$

\section{EXPERIMENTS AND DISCUSSION}

This section is devoted to show the performance of our filter compared to other speckle filters described in the background section. We carried out experiments with synthetic and real images. Synthetic experiments allow us to numerically demonstrate that the proposed filter can preserve structural information and speckle tissue details while performing filtering in regions where no medical information is present. For this purpose, two synthetic experiments are proposed. The first one is intended to show the capability of preserving tissue details and morphology, whereas the second experiment shows the performance as a speckle removal filter. Afterwards, the filters are evaluated in a practical scenario with real IVUS images in terms of robustness and relevant structural preservation. Visual results with 3D echocardiographies are also presented.

Lee, Kuan, Gamma MAP and POSRAD filters were implemented by the authors in Matlab by following rigorously the original articles. The Frost filter was implemented by Debdoot Sheet ${ }^{3}$. OSRAD code can be obtained from Karl Krissian's personal web ${ }^{4}$. SRAD code can be found at ${ }^{5}$ and DPAD code at ${ }^{6}$. The Matlab code for OBNLM can be obtained from Coupe's personal web ${ }^{7}$.

\section{A. Synthetic Images}

1) First Experiment (Kidney phantom): A B-mode US acquisition of an anatomic phantom of a human kidney was simulated by using the kidney model proposed in [38]. This phantom can be downloaded from Jensen's website ${ }^{8}$. The realistic speckle pattern was simulated by using the method proposed in [39], which does not consider any prior probabilistic description of speckle in contrast to multiplicative noise simulators typically proposed in the literature [29], [28], [30], [18]. Therefore, any bias in the results is avoided. The simulator uses the random walk model where the scattering consist of $M$ scatterers whose amplitudes are Gaussian random variables with zero mean and standard deviation depending on the intensity of the kidney map. Consequently, high intensity regions in the kidney map result in a higher echolucent response than low intensity regions.

The conventional polar US acquisition system is simulated by sampling the phantom intensity values in a polar coordinate system in combination with the random walk model that accounts for the scatterers interactions. We use 120 angular samples and 200 radial samples in order to show the resolution limits of the US acquisition system (some small structures are within a resolution cell). The number of scatterers, $M$, varies following a uniform distribution with support [5, 100] so as to cover the partially and fully speckle regime.

Finally, the envelope of the complex random walk, obtained in the polar coordinate system, is interpolated to obtain the Cartesian image. The kidney phantom and the resulting B-mode image are presented in Fig. 3.

The numerical validation was performed by using the Mean Squared Error (MSE) and the Structural Similarity Index Measure (SSIM) (the Matlab implementation of the authors [40] is available at ${ }^{9}$ ).

\footnotetext{
${ }^{3}$ http://www.mathworks.com/matlabcentral/fileexchange/35073-frosts-filter

${ }^{4}$ http://serdis.dis.ulpgc.es/ krissian/HomePage/Demos/OSRAD/OSRAD.html

${ }^{5}$ http://viva.ee.virginia.edu/downloads.html

${ }^{6} \mathrm{http}: / /$ www.mathworks.com/matlabcentral/fileexchange/36906-detail-preserving-anosotropic-diffusion-for-speckle-filtering-dpad

${ }^{7}$ https://sites.google.com/site/pierrickcoupe/softwares/denoising-for-medical-imaging/speckle-reduction/obnlm-package

${ }^{8}$ http://field-ii.dk/

${ }^{9}$ https://ece.uwaterloo.ca/ z70wang/research/ssim/
} 

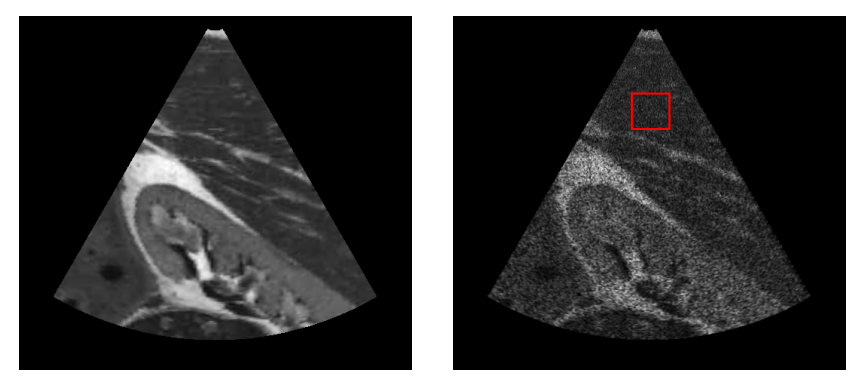

Fig. 3. Kidney phantom (left) and a B-mode simulation (right) used for the first synthetic experiment.

The results were obtained following this protocol:

1) A B-mode image of the kidney is simulated.

2) The parameters of each filter are optimized in order to minimize the MSE for the generated image.

3) A number $N_{\text {exp }}=25$ of new synthetic images are generated and the MSE and SSIM are calculated for each filter and each image. The mean $(\eta)$ and standard deviation $(\sigma)$ for these parameters are provided based on the $N_{\text {exp }}$ observations —see Table II.

Parameter Optimization: The parameters of each filter were selected according to the MSE criterion. We chose the MSE criterion instead of the SSIM because it can be related to the accuracy and precision of the true image estimation: the MSE is a more strict metric in the sense that when it converges to zero, any bias also converges to zero. Thus, a lower MSE provides a better behavior of the filter when a gold standard is provided.

An exhaustive search in the space of the parameters of each filter was performed in order to obtain the lowest MSE. In the case of Lee and Kuan filter, the only parameters to be adjusted are the variance of noise and the size of the estimation window, $W$. The MAP filter uses two parameters, the $L$ parameter and the window size, $W$. The Frost filter depends only on the size of the window.

The optimization of diffusion filters requires a more careful design. Diffusion filters are highly dependent on the diffusion step, $\Delta_{t}$, and the number of iterations, $n_{i t e r}$. In order to provide a consistent optimization, we fix both $\Delta_{t}$ and $n_{i t e r}$ for all the diffusion filters. Then, the optimal set of parameters is obtained for each filter. Finally, all filters are tested again with their optimal parameter configuration but with a unlimited number of iterations until the MSE increases. This way, filters are optimized in similar conditions and the best performance of each filter is considered. The optimization methodology can be summarized as follows:

1) We fix $\Delta_{t}$ and $n_{i t e r}$ for all diffusion filters.

2) The rest of parameters are optimized by performing an exhaustive search as in the case of classical filters. This provides an optimal configuration according to the MSE criterion.

3) Each of the filters uses its own optimal configuration until the MSE increases to set the optimal $n_{\text {iter }}$.

We select $\Delta_{t}=0.3$, as proposed in [29], to achieve stability and for the sake of comparison. The number of fixed iterations was set to $n_{\text {iter }}=20$.

Since SRAD and OSRAD filters need a region to compute the noise level, we used the same region for both as it is shown in Fig. 3. SRAD, excluding $\Delta_{t}$ and $n_{i t e r}$, only depends on the region. Once fixed, SRAD is only optimized in the third step, by selecting the best $n_{\text {iter }}$ value. DPAD filter also requires the size of the estimation window $W$. OSRAD depends on both $\sigma$, related to the structure tensor, and $c_{t a n g}$, related to the amount of diffusion along the orthogonal direction to edges.

The probabilistic model adopted for the POSRAD filter and the proposed filter is the GMM model. The number of components was set to $J=4$, since at least four types of tissues can be distinguished in the kidney image, and we experimentally confirmed that an increase in the number of components did not lead to a better description of speckle. POSRAD filter depends on both $\sigma$ and $\rho$ parameters, related to the definition of the structure tensors ( $\sigma$ is the smoothing parameter for derivatives and $\rho$ is the smoothing parameter for the structure tensor). To make our experimental analysis feasible, we restrict to the case $\sigma=\rho$. This selection considers the same smoothing kernel to detect the edges of the image and to calculate the structure tensor as used in [18]. Thus, the optimization of the proposed filter is performed as a function of $\sigma$ and the parameters from the memory mechanism, i.e., $g_{n}(y)$. In this case, $p_{\bar{c}}$ is defined as the probability of belonging to the component with the lowest mean value since structures of the kidney show a higher echolucid response. The OBNLM filter depends on three parameters, $M, \alpha$ and $h$. We considered all of them in the optimization.

The optimal configuration for all filters is shown in Table I, where the optimal number of iterations, $n_{\text {iter }}$ is also shown.

Finally, the comparison is performed by generating $N_{\text {exp }}=25$ independent samples of the B-mode kidney images with the same statistical characterization of scatterers. The filters were applied with their own optimal configurations obtained in the aforementioned steps. The results of the MSE and SSIM metrics are shown in Table II as mean plus standard deviation ${ }^{10}$ $(\eta \pm s t d)$.

\footnotetext{
${ }^{10}$ The cases where the standard deviation is lower than $10^{-4}$ are written as 0.0000 .
} 
TABLE I

OPTIMIZED PARAMETERS ACCORDING TO THE MSE CRITERION FOR THE B-MODE KIDNEY IMAGE.

\begin{tabular}{|l||l||l|}
\hline Filter & MSE & Optimal Parameters \\
\hline Lee [23] & 0.1266 & $\sigma_{u}=0.2512, W=2$ \\
\hline Kuan [25] & 0.1266 & $\sigma_{u}=0.01, W=2$ \\
\hline Gamma MAP [26] & 0.012 & Num. of Looks (L) $=4000, W=2$ \\
\hline Frost [24] & 0.0043 & $W=7$ \\
\hline SRAD [27] & 0.0164 & $\Delta_{t}=0.3, n_{\text {iter }}=80$ \\
\hline DPAD [28] & 0.00355 & $\Delta_{t}=0.3, W=2, n_{\text {iter }}=30$ \\
\hline OSRAD [29] & 0.00441 & $\Delta_{t}=0.3, \sigma=4, c_{\text {tang }}=15, n_{\text {iter }}=78$ \\
\hline POSRAD [18] & 0.00142 & $\Delta_{t}=0.3, \sigma=\rho=4, n_{\text {iter }}=26$ \\
\hline OBNLM [30] & 0.00511 & $M=6, \alpha=6, h=1$ \\
\hline Proposed & 0.00140 & $\Delta_{t}=0.3, \sigma=\rho=4, n=9, n_{\text {iter }}=31$ \\
\hline
\end{tabular}

MSE AND SSIM RESULTS FOR THE FIRST EXPERIMENT: B-MODE US IMAGE OF HUMAN KIDNEY.

\begin{tabular}{|l||c||c|}
\hline Filter & MSE & SSIM \\
\hline Noisy Image & $0.0123 \pm 0.0002$ & $0.7198 \pm 0.0014$ \\
\hline Lee [23] & $0.0130 \pm 0.0003$ & $0.7287 \pm 0.0013$ \\
\hline Kuan [25] & $0.0138 \pm 0.0003$ & $0.7254 \pm 0.0013$ \\
\hline Gamma MAP [26] & $0.0115 \pm 0.0002$ & $0.7308 \pm 0.0015$ \\
\hline Frost [24] & $0.0045 \pm 0.0002$ & $0.8497 \pm 0.0021$ \\
\hline SRAD [27] & $0.0162 \pm 0.0016$ & $0.7587 \pm 0.0136$ \\
\hline DPAD [28] & $0.0038 \pm 0.0001$ & $\mathbf{0 . 8 8 9 2} \pm 0.0016$ \\
\hline OSRAD [29] & $0.0040 \pm 0.0001$ & $0.8615 \pm 0.0016$ \\
\hline POSRAD [18] & $0.0014 \pm 0.0000$ & $0.8872 \pm 0.0021$ \\
\hline OBNLM [30] & $0.0053 \pm 0.0003$ & $0.8621 \pm 0.0035$ \\
\hline Proposed & $\mathbf{0 . 0 0 1 3} \pm 0.0000$ & $0.8814 \pm 0.0000$ \\
\hline
\end{tabular}

In Table II, the low performance of Lee, Kuan and the Gamma MAP is clearly shown for both the MSE and SSIM measures, which are practically equal to the noisy image case. This is a consequence of the blurring effect caused as the window size increases, leading to an optimal selection of the window size of $W=2$. The Frost filter obtains good results for both measures mainly due to the better description of homogeneous areas by means of local statistics and the Wiener-like behavior in the edges. Due to this poor behavior of some of the classical filters and for the sake of brevity, in what follows we just show the filtered images of the Frost filter and the non-classical filters.

Anisotropic diffusion filters perform better than the isotropic counterpart or the multiplicative based filters for both measures. This behavior was expected due to the better preservation obtained with anisotropic diffusion that prevents from the over-filtering observed in the SRAD and DPAD filters (see Fig. 4). The higher MSE of the SRAD is mainly due to the spikes obtained in regions where the instantaneous coefficient of variation is poorly estimated. In the case of DPAD, its better results for MSE and SSIM are mainly due to the effect of the background (mostly homogeneous) and the good preservation of boundaries. However, by visual inspection one can conclude that the filtered image has lost all the speckle information of the tissue and the finer details of tissue structures. This behavior makes the DPAD filter a good candidate for a preprocessing step before segmentation, though it would be unacceptable for visual inspection or diagnosis. The OSRAD filter maintains a good balance between filtering and preservation, as it is observed in the MSE and SSIM values. However, the effect of over-filtering is still appreciated, though some tissue structures are better defined than in the DPAD filtered image. The OBNLM filter reduces dramatically the noise in the background region but it enhances spurious in high echolucent regions, which results in modest numerical results for the SSIM metric (see Table II). This effect can be due to an inappropriate similarity measure (Gaussian multiplicative noise proportional to the squared root of the signal). POSRAD provides the best balance in terms of the mean of MSE and SSIM metric. Clearly, it benefits from an accurate tissue characterization as it is the case of the proposed method. However, the standard deviation of the SSIM is higher than the standard deviation provided by other —albeit worse in terms of the SSIM mean - diffusion filters. The result of the MSE measure (similar to the one obtained for the proposed method) evidences that the POSRAD method is a particular case of our proposal and that both methods optimize their parameters with respect to the MSE. This means that, in this particular case, the memory mechanism slightly increases the performance of the proposed filter compared to the simplified case of POSRAD. The better performance of POSRAD with respect to SSIM measure is mainly due to the abundance of well defined homogeneous regions in the phantom. The effect of overfiltering those regions causes a better definition of boundaries of homogeneous regions and a lower variance in the inner speckle, which increases the SSIM measure. This effect becomes clearer for DPAD, which is obviously over-filtering regions, though it obtains the highest SSIM value. In further experiments with real images, the importance of the memory mechanism will be demonstrated in terms of contrast in relevant structures and preservation of diagnostic information.

The proposed filter obtains the best result according to the MSE criterion and the third one for the SSIM value, both in mean values. The standard deviation in both cases is lower than $10^{-4}$ and therefore shown as 0 , which manifests the robustness of the method. Visually, the proposed filter preserves relevant structures within tissues (see the central part of the kidney and the high echolucent tissues above the central part). This region is better preserved than in the POSRAD case, where over-filtering can be appreciated in the internal structures. Moreover, the background tissue has been considerably filtered. 


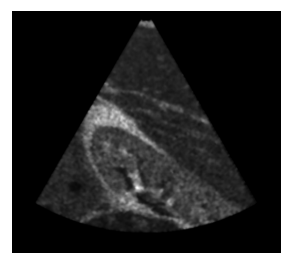

(a) Frost

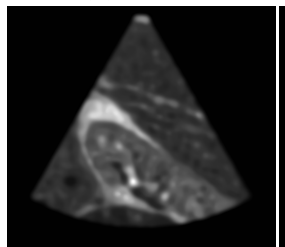

(d) OSRAD

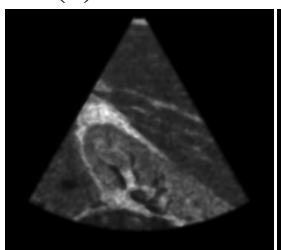

(g) Proposed

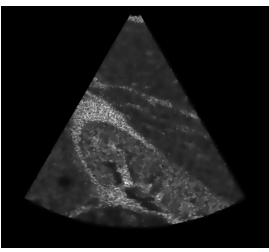

(b) SRAD

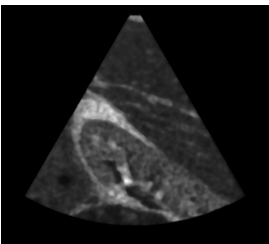

(e) POSRAD

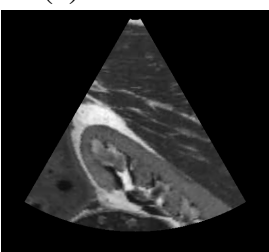

(h) Original

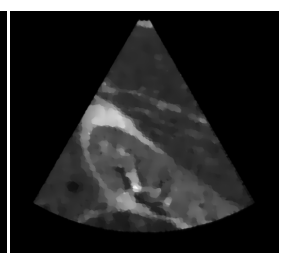

(c) DPAD

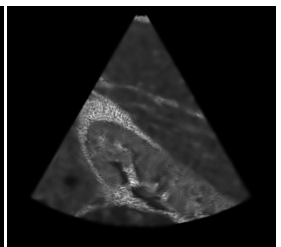

(f) OBNLM

Fig. 4. Visual comparative of different filters for the first sunthetic exneriment-B-mode_US image of human kidney.
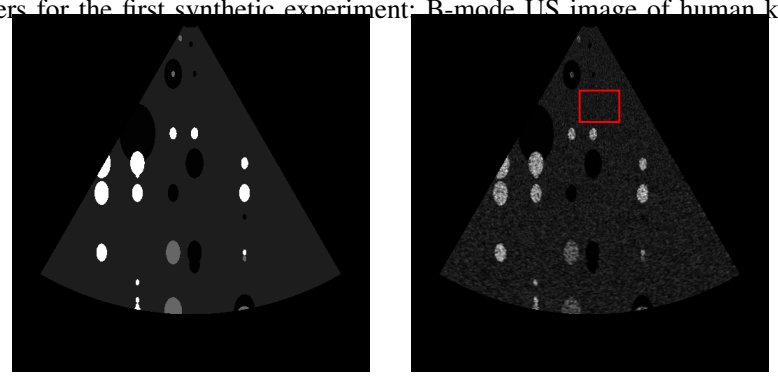

Fig. 5. Detailed cyst phantom (left) and noisy image (right) used for the second synthetic experiment.

2) Second Experiment (Detailed Cyst phantom): In this experiment we followed the methodology proposed in the OBNLM original paper [30], where some cysts were synthetically generated as hyperechoic tissue and hypoechoic tissue. We included some more responses in order to compare the performance of the speckle filters in more general scenarios. Additionally, we include small structures to account for the effects of polar sampling on them in the resulting images. We refer to this new phantom as the detailed cyst phantom.

The simulation of the speckle response of tissues was performed by using the aforementioned speckle simulator. The detailed cyst phantom and the simulated image are shown in Fig. 5, where the region for SRAD and OSRAD noise estimation is shown in Fig. 5.

The ultrasound despeckling assessment was performed by means of the index $\widehat{Q}$ used in [30] and previously defined in [41]. This index was defined to quantify the contrast between different echolucid responses and the filtering performance within them. If a phantom with $K$ classes of tissues ( $K=4$ in our experiment) is considered, the ultrasound despeckling assessment index is defined as:

$$
Q=\frac{\sum_{k=1}^{K} \sum_{l=k+1}^{K}\left(\mu_{k}-\mu_{l}\right)^{2}}{\sum_{k=1}^{K} \sigma_{k}^{2}},
$$

where $\mu_{k}$ and $\sigma_{k}^{2}$ denotes the mean and variance of $k^{t h}$ class after filtering. The difference of contrast between the classes is measured as the difference between the average of each class. In [41], the authors propose to normalize the measure $Q$ for each filter dividing by $Q_{\text {noisy }}$, which is directly obtained from the noisy image, to avoid problems with the initial resolution. We will refer to the normalized version as $\widehat{Q}$.

Parameter Optimization: We followed the same experimental protocol as in the kidney experiment by using the $\widehat{Q}$ index as the optimization criterion. The number of components for the GMM model used in POSRAD and the proposed filter is $J=4$ components, the same number as classes in the detailed cyst phantom. The proposed filter again defines $p_{\bar{c}}$ as the component of the GMM with the lowest mean value. Results obtained from the optimization process are shown in Table III.

The evaluation was performed by comparing $N_{\text {exp }}=25$ simulated speckle images. Results are shown in Table IV with the same notation as in the kidney experiment, i.e., $\eta \pm s t d$. In Fig. 6 , the filtered images for one of $N_{\text {exp }}$ experiments are shown. 
TABLE III

OPTIMIZED PARAMETERS ACCORDING TO THE $\widehat{Q}$ CRITERION FOR THE DETAILED CYST EXPERIMENT.

\begin{tabular}{|l||l||l|}
\hline Filter & $\hat{Q}$ & Optimal Parameters \\
\hline Lee [23] & 1.40 & $\sigma_{u}=0.01, W=7$ \\
\hline Kuan [25] & 1.55 & $\sigma_{u}=0.01, W=7$ \\
\hline Gamma MAP [26] & 1 & Number of Looks $(\mathrm{L})=4000, W=5$ \\
\hline Frost [24] & 2.45 & $W=7$ \\
\hline SRAD [27] & 1.03 & $\Delta_{t}=0.3, n_{\text {iter }}=91$ \\
\hline DPAD [28] & 3.14 & $\Delta_{t}=0.3, W=2, n_{\text {iter }}=29$ \\
\hline OSRAD [29] & 2.47 & $\Delta_{t}=0.3, \sigma=3.45, c_{\text {tang }}=15, n_{\text {iter }}=20$ \\
\hline POSRAD [18] & 2.37 & $\Delta_{t}=0.3, \sigma=\rho=4, n_{\text {iter }}=20$ \\
\hline OBNLM [30] & 1.53 & $M=3, \alpha=6, h=1$ \\
\hline Proposed & 3.25 & $\Delta_{t}=0.3, \sigma=\rho=3.38, n=1, n_{\text {iter }}=34$ \\
\hline
\end{tabular}

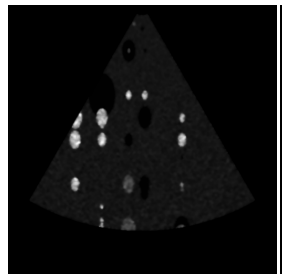

(a) Frost

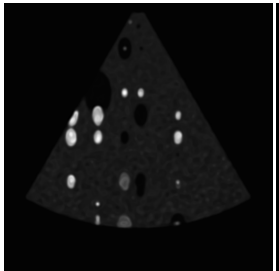

(d) OSRAD

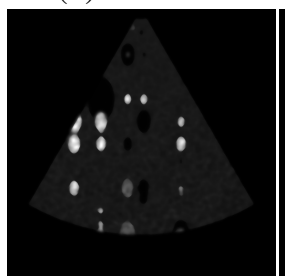

(g) Proposed

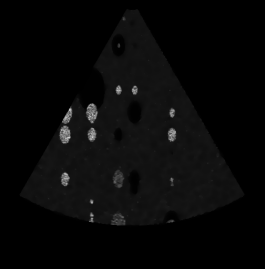

(b) SRAD

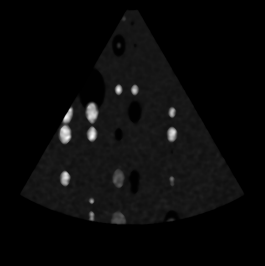

(e) POSRAD

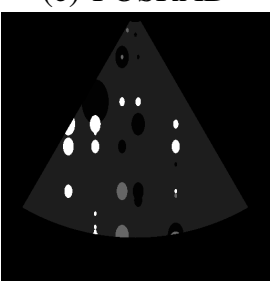

(h) Original

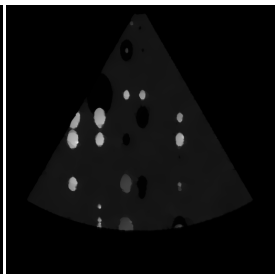

(c) DPAD

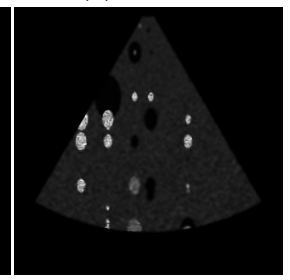

(g) OBNLM

Fig. 6. Visual comparative of the different filters for the detailed cyst experiment.

In this experiment, the Frost filter outperforms the rest of the classical filters with a competitive result $(\widehat{Q}=2.3435)$ and, consequently, this is the only one shown figure 6 . The SRAD obtains an unsatisfactory result (less than $1, Q=0.9737$ ) due to its low filtering performance within high echolucid parts, though it satisfactorily preserves the edges of those regions. The DPAD filter overcomes this problem by using the diffusion equation based on the SRAD scheme, which results in a very good figure $(\widehat{Q}=3.0838)$ due to the higher performance in the background. OSRAD and POSRAD filters are not able to improve the DPAD filter result mainly due to the higher preservation of structures in the background class. The OBNLM filter achieves a modest result $(\hat{Q}=1.4194)$, probably due to the unrealistic probabilistic model used that avoids filtering in high echolucent classes similarly as classical filters. The memory filter outperforms the rest of speckle filters with a $\widehat{Q}$ value of $\widehat{Q}=3.2145$. This result is not due to an over-filtering in the background class, as it is the case of the DPAD filter, but to the better preservation of edges between classes.

As a conclusion, the filtering performance measured by $\widehat{Q}$ seems to have an important dependence on the background. However, in the following experiments with real images, we will see that this over-filtering in background images does not necessarily lead to favorable visual results.

\section{B. Real Images}

This experiment is conceived to evaluate the performance and robustness of filters in real scenarios. Specifically, we will prove that the parameters of the state-of-the-art speckle filters need to be thoroughly tuned to produce competitive results in real images. For this purpose, we evaluate the performance of the filters, with their previously derived optimal configuration from kidney and cyst experiment, in real scenarios according to a visual criterion for clinical purposes: preservation of tissue details and speckle removal in blood areas. As previously indicated, only the Frost filter will be tested from the set of classical filters. 
TABLE IV

$\widehat{Q}$ RESULTS FOR THE SECOND EXPERIMENT: DETAILED CYST PHANTOM.

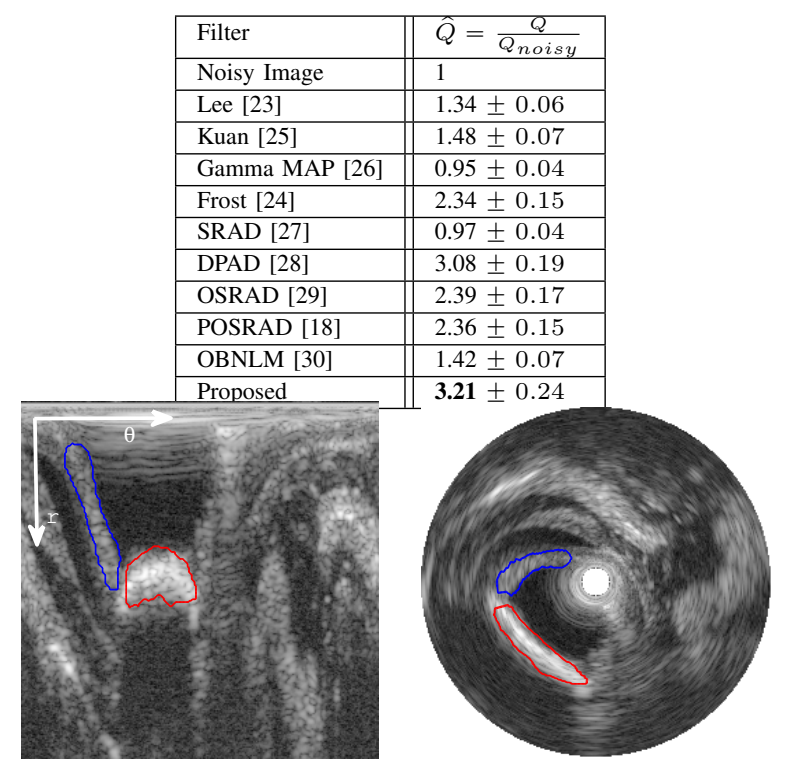

Fig. 7. IVUS image using in the experiment: (left) Polar coordinate system. (right) Cartesian coordinate system. In blue, a fibrotic plaque. In red, a calcified plaque.

1) IVUS images: The IVUS images were obtained using the Galaxy II IVUS Imaging System (Boston-Scientific) with a catheter Atlantis SR Pro $40 \mathrm{MHz}$ (Boston-Scientific). The data was sampled with $f_{s}=200 \mathrm{MHz}$ and digitalized with a 12-bit Acquiris acquisition card. The acquisition is performed using a polar coordinate system of $256 \times 256$ pixels. More information can be obtained from [5]. In this experiment, all filters were applied to the polar coordinate system before Cartesian conversion and log-compression.

The IVUS images of this data set can contain four types of tissues: calcified, fibrotic, lipidic plaques and lumen. The echogenic response of the different kinds of plaque is qualitatively known: lipidic plaques present low echolucent response, fibrotic plaques present intermediate level of echogenecity and the calcified plaque presents a higher echolucent response in combination of an acoustic shadow behind them due to the highly reflective tissue [2], [5]. The lumen region is mainly composed of blood and produces a low echolucent response. The quantitative characterization of the different plaques is especially important to understand the underlying process of the disease. Thus, the regions of the image to be filtered are those related to lumen while the echo-morphology of the aforementioned plaques should be preserved.

The IVUS image used in this experiment is shown both in polar and in Cartesian coordinates in Fig. 7. The manual segmentations (provided by experts, see [2], [5]) of a calcified and fibrotic plaque are plotted in the polar image. Both images are logarithmically compressed to enhance the contrast, but the filtering process was performed before log-compression. The region for noise estimation used in SRAD and OSRAD was located in the lumen.

a) Quantitative results: The ability for each filter to remove speckle in blood areas while preserving it in tissues is quantitatively validated by means of the contrast measure calculated as the ratio between the average intensity of segmented plaque (calcified and fibrotic), and the average intensity in the segmented lumen. For this experiment we considered three different configurations of parameters: the two configurations obtained in the aforementioned kidney and detailed cyst phantoms, and an additional set of parameters obtained by visually optimizing the configuration for each speckle filter to obtain an IVUS image where the tissue details, the morphology of the plaques and the border definition are preserved while the speckle in blood regions is suppressed. According to these criteria, each filter is run by varying the parameters in the range of the values used in the synthetic experiments to produce the best image. The configuration parameters for visual criteria are shown in Table $\mathrm{V}$ and quantitative results are reported in Table VI for the three configurations of parameters.

In all the configurations, most of filters improve the contrast in the calcified plaque as would be expected. In the case of OSRAD, the contrast is reduced mainly due to the negative influence of over-filtering. The improvement in the contrast of the fibrotic plaque is more subtle because of its lower echogenicity response. Results confirm that the proposed method considerably reduces speckle in lumen and successfully enhances plaques for every parameter configuration. This fact also evidences the higher robustness of the proposed method.

In order to show that the structures in tissues are adequately preserved, the contrast ratio can be complemented by a structural similarity measure. We use the SSIM metric to compare the filtered and unfiltered image. This comparison shows if filters avoid over-filtering and maintain the salient features and structures of plaques, which are necessary for diagnostic purposes. Results of SSIM in plaques reported in Table VII show that some filters that previously achieved a high contrast value now show poor SSIM results due to over-filtering. This is especially noticeable in the case of POSRAD, where the structure definition 
TABLE V

OPTIMIZED PARAMETERS ACCORDING TO THE VISUAL CRITERION

\begin{tabular}{|l|l|}
\hline Filter & Optimal Parameters \\
\hline Lee [23] & $\sigma_{u}=0.2, W=6$ \\
\hline Kuan [25] & $\sigma_{u}=0.2, W=6$ \\
\hline Gamma MAP [26] & Number of Looks $(\mathrm{L})=2000, W=9$ \\
\hline Frost [24] & $W=3$ \\
\hline SRAD [27] & $\Delta_{t}=0.3, n_{\text {iter }}=10$ \\
\hline DPAD [28] & $\Delta_{t}=0.3, n_{\text {iter }}=10, W=5$ \\
\hline OSRAD [29] & $\Delta_{t}=0.3, n_{\text {iter }}=18, \sigma=0.5, c_{\text {tang }}=0.05$ \\
\hline POSRAD [18] & $\Delta_{t}=0.3, n_{\text {iter }}=6, J=4 ; \sigma, \rho=0.005$ \\
\hline OBNLM [30] & $M=6, \alpha=6, h=0.05$ \\
\hline Proposed & $\Delta_{t}=0.3, n_{\text {iter }}=19, J=4 ; \sigma, \rho=0.005, n=5$ \\
\hline
\end{tabular}

CONTRAST-ENHANCEMENT RESULTS FOR IVUS IMAGES DEPENDING ON THE PARAMETERS SELECTION

\begin{tabular}{|l||c|c|c|c|c|c|}
\hline \multirow{2}{*}{ Filter } & \multicolumn{2}{|c|}{ Kidney exp. param. } & \multicolumn{2}{c|}{ Detailed cyst exp. } & \multicolumn{2}{c|}{ Visual exp. } \\
\cline { 2 - 7 } & Calc. & Fib. & Calc. & Fib. & Calc. & Fib. \\
\hline Noisy Image & 28.48 & 3.32 & 28.48 & 3.32 & 28.48 & 3.32 \\
\hline Frost [24] & 32.10 & 3.54 & 32.10 & 3.55 & 31.19 & 3.53 \\
\hline SRAD [27] & 29.40 & 3.11 & 28.73 & 3.02 & 30.30 & 3.46 \\
\hline DPAD [28] & 28.62 & 3.33 & 28.65 & 3.33 & 31.66 & 3.57 \\
\hline OSRAD [29] & 23.45 & 2.61 & 26.42 & 3.06 & 27.38 & 3.18 \\
\hline POSRAD [18] & 32.14 & 3.45 & 32.46 & 3.51 & 32.27 & 3.61 \\
\hline OBNLM [30] & 31.01 & 3.14 & 32.03 & 3.52 & 31.98 & 3.59 \\
\hline Proposed & $\mathbf{3 5 . 6 0}$ & $\mathbf{3 . 8 9}$ & $\mathbf{3 5 . 9 8}$ & $\mathbf{3 . 9 3}$ & $\mathbf{3 5 . 0 6}$ & $\mathbf{3 . 8 6}$ \\
\hline
\end{tabular}

inside the plaques has been clearly removed. Additionally, note that the filter which best preserves the speckle in plaques is our proposal, though DPAD may show the same SSIM values as our proposal but in this cases the images produced by this filter show a quasi-negligible filter effect, which is coherent with the low contrast measure results reported before.

b) Qualitative results: According to Tables VI and VII, it is clear that the proposed filter shows no significant differences between parameter configurations, in contrast to the rest of the filters. For the sake of compactness, we only show the results obtained by visual optimization. The filtered images are shown in Fig. 8. These results show that the best definition of structures is obtained for the proposed filter and the OBNLM. However, the variability of the OBNLM filter regarding its parameters seems to be important — note the variability of the SSIM measures for the three configurations of parameters - which means that some configurations suffer from over-filtering and a visualization of those images confirm this behavior. This lack of robustness is not appreciated in the proposed filter. Thus, the robustness is another important advantage of the memory filter which, in combination of the preservation performance, makes it more versatile for real scenarios.

2) 3D Echocardiographies: To complement the qualitative assessment, we show the performance of the proposed filter in real 3D echocardiographies. They were acquired with the system Philips iE33 xMATRIX by performing the 3D acquisition with the 2D array transducer X5-1. For brevity, we only show a four chamber view of the heart where the mitral valve and cavities can be easily distinguished. We also provide the resulting images for filters which achieved comparable numerical results in the previous experiments, i.e. DPAD, POSRAD and OBNLM, with the configuration provided in the cyst experiment (this one provides the best visual results for the OBNLM and POSRAD, while the proposed method and DPAD show similar results with both configurations). The images are shown in Fig. 9. Note that the best definition of structures is again obtained by the OBNLM and the proposed filter, and they also successfully remove the speckle of blood cavities. However, note that the finer structures shown in the septum and also the mitral valve are better preserved with the proposed filter. This result evidences the suitability of the proposed method to preserve relevant structures for diagnostic purposes in ultrasound images.

\section{CONClusions}

In this paper, an anisotropic diffusion speckle filter based on speckle statistics with a memory mechanism has been described. The memory mechanism is the responsible for alleviating two important drawbacks of state-of-the-art speckle filters: (1) loss of relevant tissue details due to inaccurate tissue characterization and (2) over-filtering, especially in diffusion filters, due to the progressive loss of information with the original image when the diffusion process evolves.

The memory mechanism is implemented as a DDE for the diffusion tensor, where the diffusion flux function follows a tissue selective Volterra equation. The selective behavior is related to the relaxation time $\tau$, which determines the "effective" temporal window for each tissue, avoiding the temporal average of information in regions where no relevant information is expected, e.g. blood. In an extreme case, the memory mechanism is switched off in regions with no relevant structures for clinical purposes. The memory mechanism is also based on a tensor operator $\mathcal{S}\{$.$\} which permits to reach stationary states in$ tissues where the speckle is intended to be preserved for further analysis or processing [16], [2], [4], [5]. Reaching stationary states provides a robustness that prevents from over-filtering and avoids the necessity of defining a stopping criterion. This fact is supported by the results in the experiments with real US images where no over-filtering is appreciated when compared to the rest of diffusion filters, even with a higher number of iterations.

The proposed filter achieves competitive results (when not the best) for MSE and SSIM measures. Additionally, its robustness was proved when different optimal configurations of different phantoms were tested in real images. In general, most of the 
TABLE VII

SSIM RESULTS FOR PLAQUE PRESERVATION IN IVUS IMAGES DEPENDING ON THE PARAMETERS SELECTION

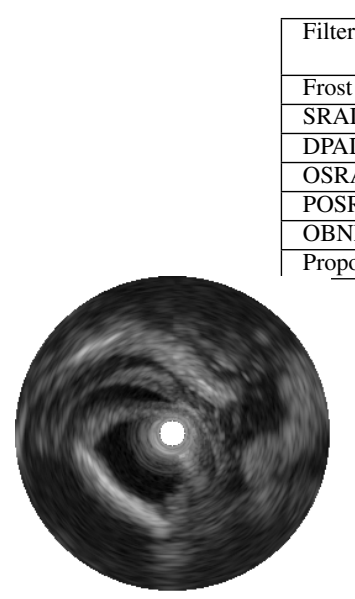

(a) Frost

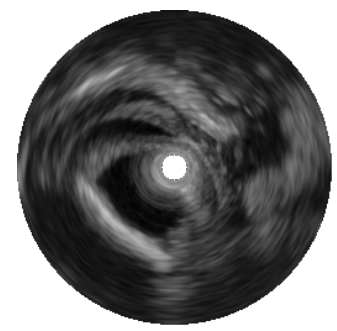

(e) POSRAD

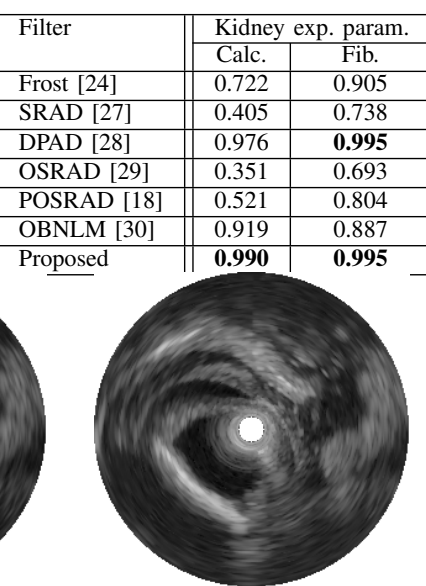

(b) SRAD

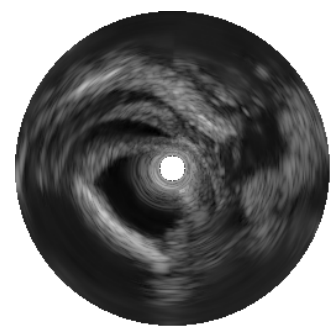

(f) OBNLM

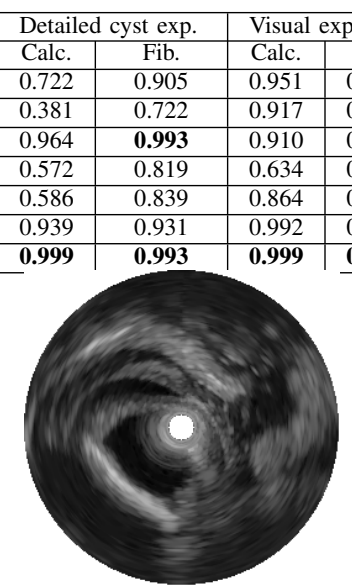

(c) DPAD

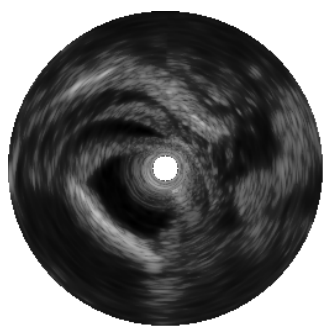

(g) Proposed

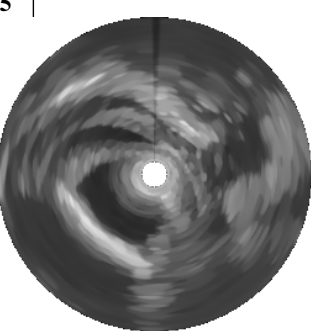

(d) OSRAD

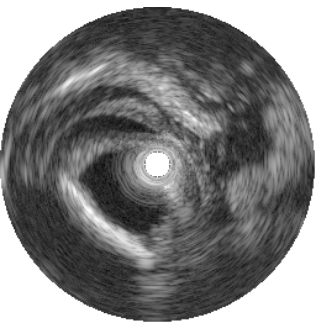

(h) Original

Fig. 8. Visual comparative of the different filters for the IVUS image: tuned parameters for tissue detail preservation and filtering in blood regions.

diffusion filters provide acceptable results for both the MSE and SSIM but, in real images, they suppress structural information of tissues. Conversely, OBNLM filter reports poor numerical results with the optimal parameters obtained with the phantom. However, it substantially improves when parameters are optimized for visual inspection. This result evidences the lack of robustness of the OBNLM which leads to a manual tunning in real scenarios. Quantitative evaluation for real images also evidences the robustness and the noticeable increase of contrast in intravascular imaging avoiding over-filtering and loss of diagnostic information.

In light of these results, we can conclude that the proposed method preserves and enhances relevant tissue details that may be useful for diagnostic purposes. It has also shown a desirable robustness which exhibits its suitability for its application in different scenarios.

\section{ACKNOWLEDGMENT}

The authors would like to thank the anonymous reviewers for their valuable comments and suggestions to improve the quality of the paper. This work was partially supported by the Junta de Castilla y León under grant VA136U13, Ministerio de Ciencia e Innovación under grant TEC2013-44194, Institute of Health Carlos III under grant PI11-0149, Consejería de Educación, Juventud y Deporte of Comunidad de Madrid and the People Programme (Marie Curie Actions) of the European Union's Seventh Framework Programme (FP7/2007-2013) under REA grant agreement n. 291820.

\section{REFERENCES}

[1] T. H. Marwick, "Measurement of strain and strain rate by echocardiography, ready for prime time?" Journal of the American College of Cardiology, vol. 47, no. 7, pp. 1313-1327, 2006.

[2] J. Seabra, F. Ciompi, O. Pujol, J. Mauri, P. Radeva, and J. Sanches, "Rayleigh mixture model for plaque characterization in intravascular ultrasound," IEEE Trans. Biomed. Eng., vol. 58, no. 5, pp. 1314-1324, 2011.

[3] J. Wilhjelm, M.-L. Gronholdt, B. Wiebe, S. Jespersen, L. Hansen, and H. Sillesen, "Quantitative analysis of ultrasound B-mode images of carotid atherosclerotic plaque: correlation with visual classification and histological examination,” IEEE Trans. Med. Imag., vol. 17, no. 6, pp. 910-922, 1998.

[4] F. Destrempes, J. Meunier, M.-F. Giroux, G. Soulez, and G. Cloutier, "Segmentation in ultrasonic B-mode images of healthy carotid arteries using mixtures of Nakagami distributions and stochastic optimization," IEEE Trans. Med. Imag., vol. 28, no. 2, pp. 215 -229, 2009.

[5] G. Vegas-Sachez-Ferrero, J. Seabra, O. Rodriguez-Leor, A. Serrano-Vida, S. Aja-Fernandez, C. Palencia, M. Martin-Fernandez, and J. Sanches, “Gamma mixture classifier for plaque detection in intravascular ultrasonic images," IEEE Trans. Ultrason., Ferroelectr., Freq. Control, vol. 61, no. 1, pp. 44-61, 2014.

[6] T. Szabo, Diagnostic Ultrasound Imaging: Inside Out, ser. Academic Press series in biomedical engineering. Elsevier Academic Press, 2004.

[7] J. G. Bosch, S. C. Mitchell, B. P. Lelieveldt, F. Nijland, O. Kamp, M. Sonka, and J. H. Reiber, "Automatic segmentation of echocardiographic sequences by active appearance motion models," IEEE Trans. Med. Imag., vol. 21, no. 11, pp. 1374-1383, 2002.

[8] A. Sarti, K. Mikula, and F. Sgallari, "Nonlinear multiscale analysis of three-dimensional echocardiographic sequences," IEEE Trans. Med. Imag., vol. 18, no. 6, pp. 453-466, 1999. 


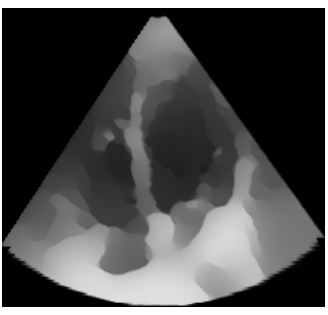

(a) DPAD

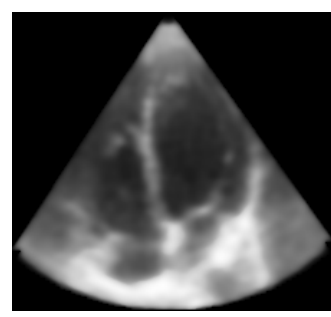

(b) POSRAD

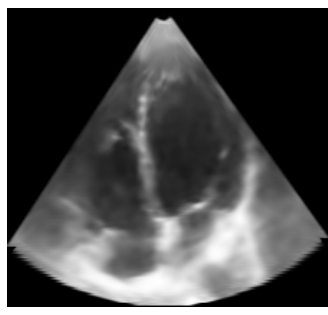

(c) OBNLM

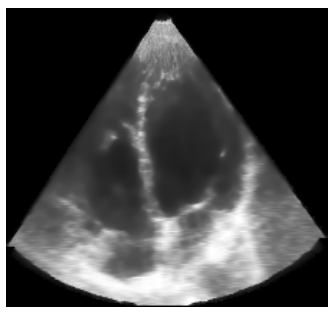

(d) Proposed

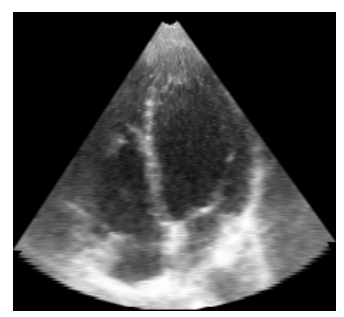

(e) Original

Fig. 9. Four chamber view of the heart of a filtered 3D echocardiography for different filters. The best definition of structures is obtained by the OBNLM and the proposed filter, which also remove successfully the speckle of blood cavities. However, finer structures observed in the septum and the mitral valve are better preserved with the proposed filter.

[9] M. Martin-Fernandez and C. Alberola-Lopez, "An approach for contour detection of human kidneys from ultrasound images using markov random fields and active contours," Medical Image Analysis, vol. 9, no. 1, pp. 1-23, 2005.

[10] D. Boukerroui, A. Baskurt, J. Noble, and O. Basset, "Segmentation of ultrasound images - multiresolution 2D and 3D algorithm based on global and local statistics," Pattern Recogn. Lett., vol. 24, no. 4-5, pp. 779-790, 2003.

[11] J. Noble and D. Boukerroui, "Ultrasound image segmentation: a survey," IEEE Trans. Med. Imag., vol. 25, no. 8, pp. 987-1010, 2006.

[12] J. W. Goodman, "Some fundamental properties of speckle," Journal of the Optical Society of America, vol. 66, no. 11, pp. 1145-1150, 1976.

[13] R. Wagner, S. Smith, J. Sandrik, and H. Lopez, "Statistics of speckle in ultrasound B-scans," IEEE Trans. Sonics Ultrason., vol. 30, no. 3, pp. 156-163, 1983.

[14] A. Myronenko, X. Song, and D. J. Sahn, "Maximum likelihood motion estimation in 3D echocardiography through non-rigid registration in spherical coordinates," in Functional Imaging and Modeling of the Heart, ser. LNCS. Springer Berlin Heidelberg, 2009, vol. 5528, pp. 427-436.

[15] R. F. Wagner, M. F. Insana, and D. G. Brown, "Unified approach to the detection and classification of speckle texture in diagnostic ultrasound," Optical Engineering, vol. 25, no. 6, pp. 25-6-738, 1986.

[16] G. Sleefe and P. Lele, "Tissue characterization based on scatterer number density estimation," IEEE Trans. Ultrason., Ferroelectr, Freq. Control, vol. 35, no. 6, pp. 749-757, 1988.

[17] V. Dutt and J. F. Greenleaf, "Ultrasound echo envelope analysis using a homodyned k distribution signal model," Ultrasonic Imaging, vol. 16, no. 4, pp. 265-287, 1994.

[18] G. Vegas-Sanchez-Ferrero, S. Aja-Fernandez, M. Martin-Fernandez, A. F. Frangi, and C. Palencia, "Probabilistic-driven oriented speckle reducing anisotropic diffusion with application to cardiac ultrasonic images," in Med Image Comput Comput Assist Interv, ser. LNCS. Berlin, Heidelberg: Springer-Verlag, 2010, pp. 518-525.

[19] P. M. Shankar, "A general statistical model for ultrasonic backscattering from tissues," IEEE Trans. Ultrason., Ferroelectr., Freq. Control, vol. 47, no. 3 , pp. $727-736,2000$.

[20] - "A compound scattering PDF for the ultrasonic echo envelope and its relationship to K and Nakagami distributions," IEEE Trans. Ultrason., Ferroelectr, Freq. Control, vol. 50, no. 3, pp. 339-343, 2003.

[21] G. Vegas-Sánchez-Ferrero, M. Martin-Fernandez, and J. Sanches, "A Gamma Mixture model for IVUS imaging," in Multi-Modality Atherosclerosis Imaging and Diagnosis. Springer New York, 2014, pp. 155-171.

[22] J. M. Thijssen and B. J. Oosterveld, "Texture in tissue echograms: speckle or information?" J. Ultrasound Med., vol. 9, no. 4, pp. 215-229, 1990.

[23] J.-S. Lee, "Digital image enhancement and noise filtering by use of local statistics," IEEE Trans. Pattern Anal. Mach. Intell, vol. 2, no. 2, pp. 165-168, 1980.

[24] V. S. Frost, J. A. Stiles, K. Shanmugan, and J. Holtzman, "A model for radar images and its application to adaptive digital filtering of multiplicative noise," IEEE Trans. Pattern Anal. Mach. Intell, vol. PAMI-4, no. 2, pp. 157-166, 1982.

[25] D. T. Kuan, A. A. Sawchuk, T. C. Strand, and P. Chavel, "Adaptive noise smoothing filter for images with signal-dependent noise," IEEE Trans. Pattern Anal. Mach. Intell, vol. 7, no. 2, pp. 165-177, 1985

[26] A. Lopes, E. Nezry, R. Touzi, and H. Laur, "Maximum a posteriori speckle filtering and first order texture models in SAR images," in Geoscience and Remote Sensing Symposium, 1990, pp. 2409-2412.

[27] Y. Yu and S. T. Acton, "Speckle reducing anisotropic diffusion," IEEE Trans. Image Process., vol. 11, no. 11, pp. 1260-1270, 2002.

[28] S. Aja-Fernández and C. Alberola-López, "On the estimation of the coefficient of variation for anisotropic diffusion speckle filtering," IEEE Trans. Image Process., vol. 15, no. 9, pp. 2694-2701, 2006.

[29] K. Krissian, C.-F. Westin, R. Kikinis, and K. G. Vosburgh, "Oriented speckle reducing anisotropic diffusion," IEEE Trans. Image Process., vol. 16, no. 5, pp. 1412-1424, 2007.

[30] P. Coupe, P. Hellier, C. Kervrann, and C. Barillot, "Nonlocal means-based speckle filtering for ultrasound images," IEEE Trans. Image Process., vol. 18, no. 10, pp. $2221-2229,2009$.

[31] J. Weickert, Anisotropic Diffusion in Image Processing. B.G Teubner, Stuttgart, Germany, 1998.

[32] P. Perona and J. Malik, "Scale-space and edge detection using anisotropic diffusion," IEEE Trans. Pattern Anal. Mach. Intell, vol. 12, no. 7, pp. 629-639, 1990.

[33] A. Buades, B. Coll, and J.-M. Morel, "A non-local algorithm for image denoising," in IEEE Computer Society Conference on Computer Vision and Pattern Recognition, vol. 2, 2005, pp. 60-65.

[34] G.-H. Cottet and M. E. Ayyadi, "A Volterra type model for image processing," IEEE Trans. Image Process., vol. 7, pp. 292-303, 1998.

[35] G. Vegas-Sánchez-Ferrero, D. Martín-Martínez, S. Aja-Fernández, and C. Palencia, "On the influence of interpolation on probabilistic models for ultrasonic images," in Proc IEEE Int Symp Biomed Imaging, 2010, pp. $292-295$.

[36] T. Moon, "The expectation-maximization algorithm," IEEE Signal Process. Mag., vol. 13, no. 6, pp. 47 -60, 1996.

[37] J. Weickert, B. T. H. Romeny, and M. A. Viergever, "Efficient and reliable schemes for nonlinear diffusion filtering," IEEE Trans. Image Process., vol. 7, no. 3, pp. 398-410, 1998.

[38] J. A. Jensen, "Field: A program for simulating ultrasound systems," in 10th Nordicbaltic Conference on Biomedical Imaging, vol. 4, Supplement 1, Part 1:351-353, 1996, pp. 351-353.

[39] C. Perreault and M.-F. Auclair-Fortier, "Speckle simulation based on B-mode echographic image acquisition model," in Fourth Canadian Conference on Computer and Robot Vision, 2007, pp. 379 -386.

[40] Z. Wang, A. C. Bovik, H. R. Sheikh, and E. P. Simoncelli, "Image quality assessment: From error visibility to structural similarity," IEEE Trans. Image Process., vol. 13, no. 4, pp. 600-612, 2004.

[41] P. C. Tay, S. T. Acton, and J. A. Hossack, "Ultrasound despeckling using an adaptive window stochastic approach," in IEEE International Conference Image Processing, 2006, pp. 2549-2552. 


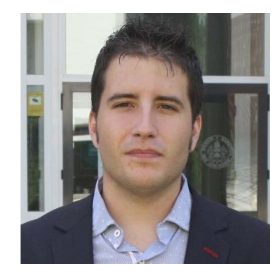

Gabriel Ramos-Llordén received the Ingeniero de Telecomunicación and the MSc in Signal Processing and Bioengineering degrees from University of Valladolid, Spain, in 2012 and 2013 respectively. Currently, he is pursuing his PhD degree at iMinds-Vision Lab, University of Antwerp, Belgium. His research interests include mathematical and signal processing methods applied to medical image problems.

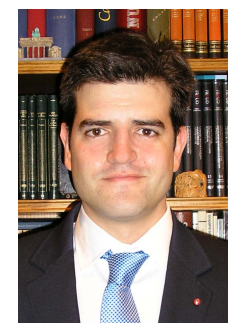

Gonzalo Vegas-Sánchez-Ferrero received the M.S. degree in telecommunication engineering from the University of Valladolid, Spain, in 2006. In 2013 he received the International Ph.D. degree and the Extraordinary Doctorate Award of University of Valladolid. $\mathrm{He}$ also was finalist of the best Ph.D. Thesis on information technologies and telecommunications of the Official College of Telecommunications Engineering of Spain. He was a Visiting Researcher with the Center for Computational Imaging \& Simulation Technologies in Biomedicine, Barcelona, Spain; Instituto Superior Tecnico, Lisboa, Portugal; the Athena Research Team, INRIA, Sophia-Antipolis, France; and the Thoraxcenter, Erasmus MC, Rotterdam. He is a coeditor and reviewer of several scientific journals. His research interests include statistical and mathematical methods for image and signal processing. Dr. Vegas-Sánchez-Ferrero was the recipient of an FPI grant from the Spanish Government and he is currently an M+VISION Fellow of the Madrid-MIT Consortium.

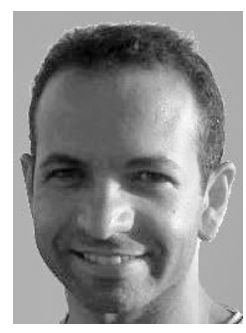

Marcos Martin-Fernandez received the Ingeniero de Telecomunicación and the Ph.D. degrees from the University of Valladolid, Spain, in 1995 and 2002, respectively. He is an Associate Professor at the ETSI Telecomunicación, University of Valladolid, where he is currently teaching and supervising several masters degree and Ph.D. students. From March 2004 to March 2005, he was a Visiting Assistant Professor of Radiology at the Laboratory of Mathematics in Imaging (Surgical Planning Laboratory, Harvard Medical School, Boston, MA) thanks to a Fulbright fellowship grant. His research interests are statistical methods for signal and image segmentation and filtering in multidimensional signal processing. He also works in the application of mathematical methods to solve image processing problems. He is also researching in the fields of magnetic resonance imaging, ultrasonic imaging, and electrophysiological signal analysis and synthesis. He is associated with the Laboratory of Image Processing (LPI) at the University ofValladolid, where he is currently performing his research. He has 120 published papers in scientific journals and conferences.

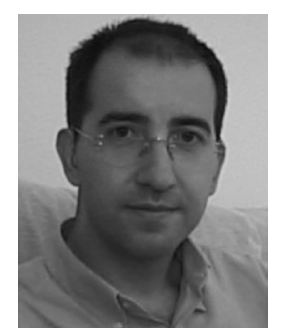

Carlos Alberola-López received the Ingeniero de Telecomunicación and Ph.D. degrees from the Polytechnic University of Madrid, Madrid, Spain, in 1992 and 1996, respectively. In 1997, he was a Visiting Scientist with Thayer School of Engineering, Dartmouth College, Hanover, NH. He is currently a Professor with the E.T.S. Ingenieros de Telecomunicación, University of Valladolid, Valladolid, Spain, where he is also the Head of the Laboratory of Image Processing. He is a coeditor and/or reviewer of several scientific journals and main conferences, and he is a Consultant of the Spanish Government for the evaluation of research initiatives. He is a coauthor of several book chapters and more than 100 journal and conference papers. His research interests include statistical signal and image processing applications, particularly focused on, but not limited to, the field of bioengineering.

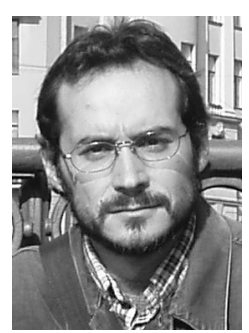

Santiago Aja-Fernández received the Ingeniero de Telecomunicación and $\mathrm{PhD}$ degrees from the University of Valladolid, Spain, in 1999 and 2003, respectively. He is an assistant professor with the E.T.S. Ingenieros de Telecomunicación, University of Valladolid, where he is also with the Laboratory of Image Processing (LPI). His research interests include medical image analysis and processing. He received the Fulbright Scholarship for a one-year stay as a research fellow with the Laboratory of Mathematics in Imaging, Brigham, and Women's Hospital, Harvard Medical School, Boston. 